\title{
Calcium- and Metabolic State-Dependent Modulation of the Voltage-Dependent Kv2.1 Channel Regulates Neuronal Excitability in Response to Ischemia
}

\author{
Hiroaki Misonou, Durga P. Mohapatra, Milena Menegola, and James S. Trimmer \\ Department of Pharmacology, School of Medicine, University of California, Davis, Davis, California 95616
}

\begin{abstract}
Ischemic stroke is often accompanied by neuronal hyperexcitability (i.e., seizures), which aggravates brain damage. Therefore, suppressing stroke-induced hyperexcitability and associated excitoxicity is a major focus of treatment for ischemic insults. Both ATP-dependent and $\mathrm{Ca}^{2+}$-activated $\mathrm{K}^{+}$channels have been implicated in protective mechanisms to suppress ischemia-induced hyperexcitability. Here we provide evidence that the localization and function of Kv2.1, the major somatodendritic delayed rectifier voltage-dependent $\mathrm{K}^{+}$ channel in central neurons, is regulated by hypoxia/ischemia-induced changes in metabolic state and intracellular $\mathrm{Ca}^{2+}$ levels. Hypoxia/ ischemia in rat brain induced a dramatic dephosphorylation of Kv2.1 and the translocation of surface Kv2.1 from clusters to a uniform localization. In cultured rat hippocampal neurons, chemical ischemia (CI) elicited a similar dephosphorylation and translocation of $\mathrm{Kv} 2.1$. These events were reversible and were mediated by $\mathrm{Ca}^{2+}$ release from intracellular stores and calcineurin-mediated Kv2.1 dephosphorylation. CI also induced a hyperpolarizing shift in the voltage-dependent activation of neuronal delayed rectifier currents $\left(I_{\mathrm{K}}\right)$, leading to enhanced $I_{\mathrm{K}}$ and suppressed neuronal excitability. The $I_{\mathrm{K}}$ blocker tetraethylammonium reversed the ischemia-induced suppression of excitability and aggravated ischemic neuronal damage. Our results show that $\mathrm{Kv} 2.1 \mathrm{can}$ act as a novel $\mathrm{Ca}^{2+}$ - and metabolic state-sensitive $\mathrm{K}^{+}$channel and suggest that dynamic modulation of $I_{\mathrm{K}} / \mathrm{Kv} 2.1$ in response to hypoxia/ischemia suppresses neuronal excitability and could confer neuroprotection in response to brief ischemic insults.
\end{abstract}

Key words: channel; epilepsy; hypoxia; ion channels; hippocampus; neurons; neuroprotection

\section{Introduction}

Hypoxia is the most common cause of neonatal seizures and a risk factor for the later development of epilepsy. In adults, ischemic attack is often accompanied by seizures (Varelas and Mirski, 2001), and the resulting hyperexcitability may aggravate brain damage from ischemic stroke. Indeed, there are many similarities between ischemia and epilepsy regarding brain-damaging mechanisms (Leker and Neufeld, 2003). In each of these pathological conditions, an acute membrane depolarization caused by voltage- and ligand-gated ion channels results in neuronal hyperexcitability, intracellular $\mathrm{Ca}^{2+}\left(\left[\mathrm{Ca}^{2+}\right]_{\mathrm{i}}\right)$ overload, and neuronal damage, together referred to as excitotoxicity (Lee et al., 1999). On the basis of these similarities, antiepileptic drugs have been tested as neuroprotective agents in animal models of stroke, and some, especially channel blockers, have shown promising effects (Calabresi et al., 2003). Therefore, suppressing neuronal hyper-

Received April 7, 2005; revised Sept. 26, 2005; accepted 0ct. 15, 2005.

This research was supported by National Institutes of Health/National Institute of Neurological Disorders and Stroke Grant NS42225 (J.S.T.), The Epilepsy Foundation (H.M.), and American Heart Association Grant 0565091Y (H.M.). Confocal imaging was conducted in a facility constructed with support from National Center for Research Resources, National Institutes of Health Research Facilities Improvement Program Grant Number C06 RR-12088-01. We thank Drs. R. Adamson and E. Yamoah for help with $\mathrm{Ca}^{2+}$ imaging and Drs. K. Rhodes, J. Antognini, M. O'Donnell, and D. E. Logothetis for helpful discussion.

Correspondence should be addressed to Hiroaki Misonou, Department of Pharmacology, School of Medicine, One Shields Avenue, University of California, Davis, Davis, CA 95616. E-mail: hmisono@ucdavis.edu.

DOI:10.1523/JNEUROSCI.3370-05.2005

Copyright $\odot 2005$ Society for Neuroscience $\quad$ 0270-6474/05/2511184-10\$15.00/0 excitability after ischemic insults might be a fertile therapeutic target for stroke.

Voltage-dependent $\mathrm{K}^{+}(\mathrm{Kv})$ channels are potent suppressors of neuronal excitability and represent key candidates for physiological and therapeutic modulation of the neuronal hyperexcitability that occurs in hypoxic/ischemic and epileptic patients. Delayed rectifier $\mathrm{Kv}$ currents $\left(I_{\mathrm{K}}\right)$ are important in regulating somatodendritic excitability in hippocampal and cortical pyramidal neurons (Bekkers, 2000; Du et al., 2000; Korngreen and Sakmann, 2000). In these neurons, the Kv2.1 channel is the major component of somatodendritic $I_{\mathrm{K}}$ (Martina et al., 1998; Murakoshi and Trimmer, 1999; Du et al., 2000; Malin and Nerbonne, 2002; Pal et al., 2003). Interestingly, many studies of somatodendritic $I_{K}$, and of Kv2.1, in pyramidal neurons suggest a function in regulating excitability and $\mathrm{Ca}^{2+}$ influx during periods of repetitive high-frequency firing (Colbert and Pan, 1999; Bekkers, 2000; Du et al., 2000; Kang et al., 2000; Korngreen and Sakmann, 2000; Misonou et al., 2004) rather than in a more classical role in action potential repolarization. We found previously that increased neuronal activity induced by seizures or glutamate stimulation changes Kv2.1 phosphorylation state and localization and yields increased amplitude of hippocampal neuron $I_{\mathrm{K}} / \mathrm{Kv} 2.1$ attributable to hyperpolarizing shifts in voltage-dependent activation (Misonou et al., 2004). Such enhanced activity of Kv2.1 under conditions of hyperexcitability is predicted to provide homeostatic suppression of neuronal activity (Surmeier and Foehring, 2004; Misonou et al., 2005). 
We present here the surprising finding that in vivo, brief $\mathrm{CO}_{2}$ anesthesia results in dephosphorylation of brain Kv2.1 and dispersion of somatodendritic Kv2.1 clusters in neurons throughout the brain. Short periods of hypoxia/ischemia can induce similar modulation of Kv2.1 channels in cultured hippocampal neurons by inducing intracellular $\mathrm{Ca}^{2+}$ release, presumably from mitochondria, and calcineurin-mediated Kv2.1 dephosphorylation. The ATP- and $\mathrm{Ca}^{2+}$-mediated modulation of $\mathrm{Kv} 2.1$ is associated with increased amplitude of neuronal $I_{\mathrm{K}}$ attributable to a hyperpolarizing shift in the voltage dependence of activation and a hypoxia/ischemia-induced suppression of neuronal excitability. Together with our previous findings of Kv2.1 modulation in seizure animals (Misonou et al., 2004), we suggest that phosphorylation-dependent modulation of the abundant somatodendritic Kv2.1 channel may be a general mechanism to suppress pathological hyperexcitability of central neurons.

\section{Materials and Methods}

Preparation of hypoxic/ischemic animals. All animal use procedures were performed in strict accordance with the Guide for the Care and Use of Laboratory Animals described by the National Institutes of Health. Rats were subjected to $\mathrm{CO}_{2}$ inhalation in a closed chamber connected to a $\mathrm{CO}_{2}$ gas cylinder. Some animals were anesthetized by inhalation of diethyl ether or isoflurane in a closed chamber or by intraperitoneal injection of pentobarbital $(50 \mathrm{mg} / \mathrm{kg})$ or a mixture of ketamine and xylazine. Animals were then either decapitated to remove the brains or perfused with $4 \%$ paraformaldehyde for immunohistochemistry. To induce complete global ischemia, decapitated heads were incubated for $4 \mathrm{~min}$ at room temperature $\left(\sim 25^{\circ} \mathrm{C}\right)$. For immunoblotting, a crude whole-brain membrane fraction was prepared from these animal brains, and proteins were analyzed on immunoblotting as described previously (Misonou et al., 2004). For immunohistochemical analyses, sagittal brain sections (each $40 \mu \mathrm{m}$ thick) were prepared from perfusion-fixed animals (Rhodes et al., 2004). The sections were blocked with $10 \%$ goat serum and then stained with a rabbit anti-Kv2.1 antibody (Trimmer, 1991) using Alexaconjugated secondary antibodies (Invitrogen, Carlsbad, CA) as described previously (Rhodes et al., 2004). Fluorescent images were taken with a 24-bit color digital camera installed on a Zeiss (Oberkochen, Germany) Axiovert 200M microscope with a $63 \times, 1.3$ numerical aperture lens and a grating imaging system, Apotome, using Axiovision software.

Materials. Unless stated otherwise, all reagents were obtained from Sigma (St. Louis, MO) or Roche (Indianapolis, IN).

Neuronal culture. Dissociated cultures of embryonic rat hippocampal neurons were prepared as previously described (Misonou et al., 2004).

Biochemical analysis of neuronal proteins. Samples for biochemical analyses were prepared from neuronal cultures as described previously (Misonou et al., 2004). Briefly, neurons were washed twice with HBSS and incubated with drugs as indicated in the figure legends. The cells were then washed twice with ice-cold Locke's solution (in mM: $154 \mathrm{NaCl}$, $5.6 \mathrm{KCl}, 2.3 \mathrm{CaCl}_{2}, 1 \mathrm{MgCl}_{2}, 5$ glucose, and 5 HEPES, pH 7.4), harvested, and centrifuged at $12,000 \times \mathrm{g}$ for $30 \mathrm{~min}$ at $4^{\circ} \mathrm{C}$. The pellets were extracted by adding sample buffer (2\% SDS, 1 mM EDTA, $10 \%$ glycerol, $0.001 \%$ bromophenol blue, $5 \% \beta$-mercaptoethanol, and $62.5 \mathrm{~mm}$ Tris$\mathrm{HCl}, \mathrm{pH} 6.8$ ) and sonicating briefly. Rat brain membranes were prepared and treated with purified phosphatases as described previously (Misonou et al., 2004). Proteins were separated on 7.5\% SDS-PAGE, transferred to nitrocellulose membrane, and immunoblotted with anti-Kv2.1 mouse monoclonal antibody K89/41 (Antonucci et al., 2001) or anti-Kv4.2 mouse monoclonal antibody K57/1 (Rhodes et al., 2004). The blots were incubated with HRP-conjugated secondary antibodies (MP Biomedicals, Irvine, CA) followed by enhanced chemiluminescence reagent (PerkinElmer, Wellesley, MA). Immunoreactive bands were visualized by exposing the blot to $\mathrm{x}$-ray film. Because the Kv2.1 population of relative electrophoretic mobility $\left(\mathrm{M}_{\mathrm{r}}\right) \approx 125 \mathrm{kDa}$ represents the major constitutively phosphorylated form of neuronal Kv2.1 (Murakoshi et al., 1997), here we quantified the relative amounts of this species as a measure of Kv2.1 phosphorylation state (Misonou et al., 2004). Immunore- active bands were quantified after scanning using NIH ImageJ software (http://rsb.info.nih.gov/ij/) with the gel plotting macro. Quantitation was performed on samples from at least three different cultures.

ATP assay. Neurons were incubated with $5 \mathrm{~mm}$ 2-deoxy-D-glucose and $5 \mathrm{~mm}$ sodium azide for $5-15 \mathrm{~min}$ to induce chemical ischemia (CI), washed twice with ice-cold Locke's solution, and harvested in lysis buffer ( $1 \%$ Triton $\mathrm{X}-100$ and $10 \mathrm{~mm}$ Tris- $\mathrm{HCl}, \mathrm{pH}$ 8.0). As a control, neurons were incubated without the CI reagents, but the reagents were added immediately before the wash step. The suspension was centrifuged at $16,000 \times g$ for $10 \mathrm{~min}$ at $4^{\circ} \mathrm{C}$ to remove insoluble materials. The resultant lysates were analyzed for ATP levels by using an ATP assay kit based on a luciferin-luciferase method.

Immunofluorescence staining of neurons. Neurons were incubated with drugs as indicated in the figure legends and then fixed and stained with K89/41 and anti-microtubule-associated protein-2 (MAP-2) mouse monoclonal antibodies and Alexa-conjugated isotype-specific secondary antibodies (Invitrogen) as described previously (Misonou et al., 2004). Fluorescent images were taken with a 24-bit color digital camera installed on a Zeiss Axioskop 2 microscope with a $63 \times, 1.3$ numerical aperture lens, using Axiovision software. Images were transferred to Adobe PhotoShop software (Adobe Systems, San Jose, CA) as JPEG files. Quantitative analyses of Kv2.1 clustering were done using raw images. The percentage of cells exhibiting diffuse Kv2.1 staining was determined by collecting images from 100 cells from each of two independent experiments (Lim et al., 2000).

$\mathrm{Ca}^{2+}$ imaging. Neurons were washed twice with HBSS supplemented with 10 mm HEPES, pH 7.4, and then loaded with $5 \mu \mathrm{M}$ Fluo-4-AM (Invitrogen) for $5 \mathrm{~min}$ at room temperature $\left(23-25^{\circ} \mathrm{C}\right)$. After washing out excess dye, cells were further incubated for $15 \mathrm{~min}$ to allow deesterification of AM ester. Neurons were imaged at $1 \mathrm{~Hz}$ using confocal laser scanning microscopy (Zeiss LSM510) with argon laser $(488 \mathrm{~nm})$ and a $40 \times, 1.3$ numerical aperture lens. Fluorescence intensity was measured on the NIH ImageJ software (http://rsb.info.nih.gov/ij/). Values were expressed as ratio of fluorescence intensity $(F)$ against the basal intensity $\left(F_{0}\right), \Delta F / F_{0}$. For the analyses of spontaneous $\mathrm{Ca}^{2+}$ transients, frequencies of $\mathrm{Ca}^{2+}$ transients with $\Delta F / F_{0}>0.2$ were measured and expressed as percentages of the values in control. Alternatively, total $\mathrm{Ca}^{2+}$ loads by $\mathrm{Ca}^{2+}$ transients were measured by integrating traces of $\mathrm{Ca}^{2+}$ signal for $2 \mathrm{~min}$.

Electrophysiology. $I_{\mathrm{K}}$ current recordings were made with the whole-cell voltage-clamp configuration. Patch pipettes were pulled from borosilicate glass tubing, heat-polished at the tip to give a resistance of 1-2 $\mathrm{M} \Omega$ when filled with the pipette solution (in mM: $140 \mathrm{KCl}, 5 \mathrm{EGTA}, 2 \mathrm{MgCl}_{2}$, 10 glucose, and 10 HEPES, $\mathrm{pH}$ 7.3). Currents were recorded with a patchclamp amplifier (EPC-10; HEKA Elektronik, Lambrecht/Pfalz, Germany), sampled at $10 \mathrm{kHz}$, and filtered at $2 \mathrm{kHz}$ by a digital Bessel filter. All currents were capacitance and series resistance compensated and leak subtracted by standard $P / n$ procedure. External solution contained 0.1 $\mu \mathrm{M}$ tetrodotoxin (TTX), $140 \mathrm{~mm} \mathrm{NaCl}, 5 \mathrm{~mm} \mathrm{KCl}, 2 \mathrm{~mm} \mathrm{CaCl} 2,2 \mathrm{~mm}$ $\mathrm{MgCl}_{2}, 10 \mathrm{~mm}$ glucose, and $10 \mathrm{~mm}$ HEPES, pH 7.3. CI (5 mm 2-deoxyD-glucose and $5 \mathrm{~mm}$ sodium azide) was applied to neurons for $15 \mathrm{~min}$ using a polytetrafluorethylene-glass multiple-barrel perfusion system. Currents were recorded from neurons before and after induction of CI. All recordings were performed at room temperature $\left(23-25^{\circ} \mathrm{C}\right)$. The membrane potential was held at $-100 \mathrm{mV}$ and depolarized from the holding potential of $-100 \mathrm{mV}$ to voltages between -90 and $+80 \mathrm{mV}$ in $10 \mathrm{mV}$ increments for $200 \mathrm{~ms}$. Before the start of the test depolarization, a single prepulse to $-10 \mathrm{mV}$ was given for $30 \mathrm{~ms}$ to inactivate majority of the transient outward $\mathrm{K}^{+}$currents. Peak outward sustained $\mathrm{K}^{+}$currents (I) were measured $100 \mathrm{~ms}$ into the pulse at each depolarizing potential and were converted to conductance $(G)$ using the equation, $G=I /(V-$ $\left.E_{\mathrm{k}}\right)$. The Nernst $\mathrm{K}^{+}$equilibrium potential $E_{\mathrm{K}}$ was calculated as $-84 \mathrm{mV}$. The normalized conductances were plotted against the test potential $(V)$ and fitted to a single Boltzmann equation $G=G_{\max } /(1+\exp [-(V-$ $\left.\left.V_{1 / 2}\right) / k\right]$ ), where $G_{\max }$ is the maximum conductance, $V_{1 / 2}$ is the test potential at which the $I_{\mathrm{K}}$ channels have a half-maximal conductance, and $k$ represents the slope of the activation curve.

Viability assay. Viability of neurons was assessed using the LIVE/ DEAD assay kit (Invitrogen). Cells were incubated with CI reagents in 

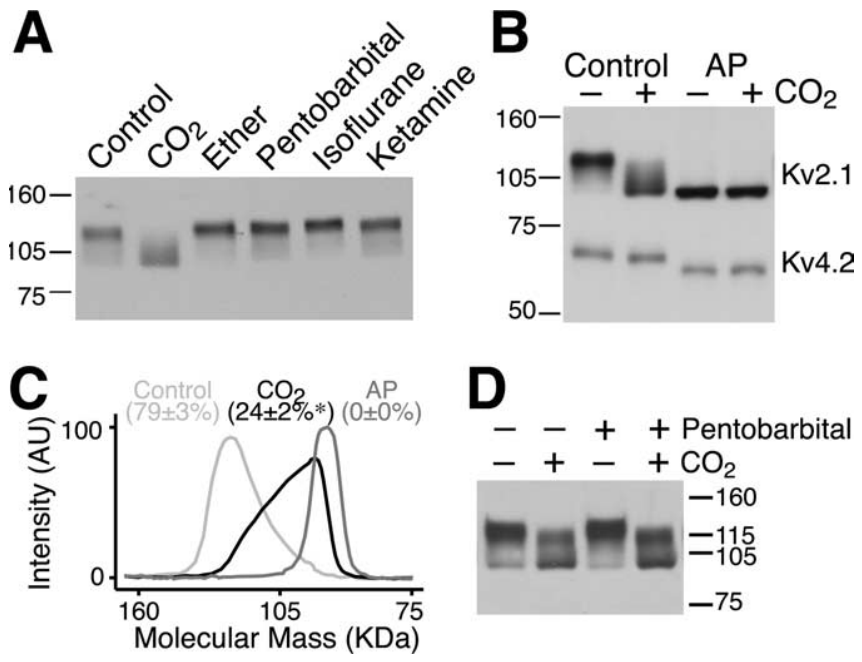

Figure 1. $\mathrm{CO}_{2}$ inhalation induces dephosphorylation of Kv2.1 in vivo. $\boldsymbol{A}$, Effects of different anesthetics on the phosphorylation state of Kv2.1 in vivo. Animals were anesthetized by $2.5 \mathrm{~min}$ inhalation of $\mathrm{CO}_{2}$, diethyl ether (Ether), isoflurane, or by intraperitoneal injection of $50 \mathrm{mg} / \mathrm{kg}$ pentobarbital, or $24 \mathrm{mg} / \mathrm{kg}$ ketamine and $3.2 \mathrm{mg} / \mathrm{kg}$ xylazine (Ketamine) and were then decapitated to prepare brain membrane fractions. Proteins were separated by SDS-PAGE and analyzed for Kv2.1 by immunoblotting. Numbers on the left indicate the mobility of molecular weight standards in kilodaltons. $\boldsymbol{B}$, Crude membranes were prepared from brains of control and $\mathrm{CO}_{2}$-treated animals and incubated without (control) or with $0.1 \mathrm{U} / \mathrm{ml} \mathrm{AP}$. Proteins were analyzed by immunoblotting for Kv2.1 and Kv4.2. C, Line scan analysis of immunoblots showing dramatic dephosphorylation of $\mathrm{Kv} 2.1$ in $\mathrm{CO}_{2}$-treated animals. Bands of total and phosphorylated $\left(M_{r}\right.$ of $25 \mathrm{kDa}$ ) Kv2.1 were quantified. Numbers below the labeling indicate the amount of phosphorylated Kv2.1 as a percentage of total Kv2.1 level and are the mean \pm SEM from three independent experiments. ${ }^{*} p<0.001$ compared with control. AU, Arbitrary units. D, Effects of pentobarbital on $\mathrm{CO}_{2}$-induced $\mathrm{Kv} 2.1$ dephosphorylation. Animals were injected with vehicle or $50 \mathrm{mg} / \mathrm{kg}$ sodium pentobarbital and then treated with $\mathrm{CO}_{2}$ for $2 \mathrm{~min}$. Crude brain membranes were prepared and analyzed by immunoblotting for Kv2.1.

HBSS for 10 min, washed twice with HBSS, and incubated with HBSS in the presence or absence of $5 \mathrm{~mm}$ tetraethylammonium (TEA) or $10 \mathrm{~nm}$ iberiotoxin for $1 \mathrm{~h}$. Cells were then washed twice with HBSS and brought back to the normal culture condition. Cell viability was assessed after $24 \mathrm{~h}$ incubation. Numbers of live cells (calcein-positive cells, in green) and dead cells (ethidium homodimer-1 positive cells, in red) were counted in five areas $\left(\sim 7.5 \mathrm{~mm}^{2}\right.$ each) imaged with a $2.5 \times$ objective in three different cultures, and the viability was expressed as percentage viable cells, relative to control (untreated) cultures. Minor populations of nonneuronal cells in these relatively pure hippocampal cultures were excluded from the count.

Statistical analyses. All data are presented as the mean \pm SEM. Normal data were compared by a two-sided $t$ test. A $t$ value of $p<0.05$ was considered to be statistically significant.

\section{Results}

\section{$\mathrm{CO}_{2}$ anesthesia rapidly induces dephosphorylation of rat} brain Kv2.1

The delayed rectifier Kv2.1 channel $\alpha$ subunit is a sixtransmembrane segment polypeptide with large cytoplasmic $\mathrm{N}$ and $\mathrm{C}$ termini that contain more than 60 candidate phosphorylation sites. On SDS-polyacrylamide gels, the major forms of Kv2.1 in rat brain and cultured embryonic rat hippocampal neurons exhibit substantially higher $\mathrm{M}_{\mathrm{r}}(\approx 125$ and $\approx 115 \mathrm{kDa})$ than predicted from the deduced Kv2.1 primary sequence $(95.3 \mathrm{kDa})$. In vitro alkaline phosphatase (AP) treatment of extracts prepared from either brain or cultured neurons shifts the $\mathrm{M}_{\mathrm{r}}$ of Kv2.1 to $\approx 100 \mathrm{kDa}$ (Misonou et al., 2004) (Fig. 1 B), suggesting that Kv2.1 is constitutively and highly phosphorylated in both brain and cultured hippocampal neurons.
In response to revised institutional guidelines for animal care, we began to use $\mathrm{CO}_{2}$ gas to deeply anesthetize animals before they were decapitated. However, we found that Kv2.1 in samples prepared from the brains of $\mathrm{CO}_{2}$-anesthetized rats exhibited an altered $\mathrm{M}_{\mathrm{r}}(\approx 100 \mathrm{kDa})$ that was similar to that of Kv2.1 in APtreated samples, and distinct from the mobility of Kv2.1 $(\approx 125$ $\mathrm{kDa})$ in previous samples prepared from animals decapitated without $\mathrm{CO}_{2}$ anesthesia (Fig. $1 \mathrm{~A}$ ). When we examined the effects of various forms of anesthesia on the phosphorylation state of Kv2.1 in vivo, we found that $\mathrm{CO}_{2}$ was unique in causing this significant shift in the $\mathrm{M}_{\mathrm{r}}$ of Kv2.1 (Fig. 1A). The differences in $\mathrm{M}_{\mathrm{r}}$ of $\mathrm{Kv} 2.1$ in samples from control and $\mathrm{CO}_{2}$-treated animals were eliminated by AP treatment (Fig. $1 B, C$ ), showing that they were attributable solely to changes in Kv2.1 phosphorylation state. The $\mathrm{M}_{\mathrm{r}}$ of two related rat brain $\mathrm{Kv}$ channel $\alpha$ subunits, Kv4.2 (Fig. $1 B$ ) and Kv1.1 (data not shown), which also undergo significant $\mathrm{M}_{\mathrm{r}}$ shifts during in vitro AP treatment, were not altered by the brief $\mathrm{CO}_{2}$ anesthesia. We have shown previously that kainate-induced seizures elicit dephosphorylation of Kv2.1 in vivo (Misonou et al., 2004). That the $\mathrm{CO}_{2}$-induced dephosphorylation of Kv2.1 shown here was independent of $\mathrm{CO}_{2}$-induced seizure activity was shown by the fact that the levels of phosphorylated Kv2.1 were not significantly different ( $p=0.09 ; n=3$ ) between rats subjected to $\mathrm{CO}_{2}$ inhalation in the absence or presence of deep pentobarbital anesthesia (Fig. 1D).

\section{$\mathrm{CO}_{2}$ anesthesia dramatically changes the subcellular localization of neuronal Kv2.1 in vivo}

Kv2.1 is abundantly expressed in virtually all mammalian brain neurons (Trimmer and Rhodes, 2004) and is present in large somatodendritic clusters (Trimmer, 1991) that disperse in response to kainate-induced seizures (Misonou et al., 2004). To address whether $\mathrm{CO}_{2}$ anesthesia altered Kv2.1 clustering, pentobarbital-anesthetized rats were subjected to brief $(2 \mathrm{~min})$ $\mathrm{CO}_{2}$ inhalation and then rapidly perfused with paraformaldehyde fixative to minimize postmortem ischemic effects. In brains of control (pentobarbital anesthetized) rats, immunofluorescence staining for Kv2.1 was present as distinct surface clusters on somata and proximal dendrites of neurons in hippocampus and subiculum (Fig. 2), as well as cerebral cortex, thalamus, hypothalamus, and striatum (data not shown). In brains from $\mathrm{CO}_{2}$ treated animals, these high-density clusters of Kv2.1 were dispersed such that Kv2.1 staining was now uniform on the surface membrane in virtually all neurons in the hippocampal formation (Fig. 2) and in all other brain regions examined (data not shown). In contrast, the localization of other neuronal $\mathrm{K}^{+}$channels (Kv4.2, Kv1.4, and BK/Slo) was not significantly altered in the brains of the $\mathrm{CO}_{2}$-treated animals (data not shown). Optical sectioning (Lanni and Wilson, 2000) of the Kv2.1-stained brain sections revealed that Kv2.1 remained in the neuronal membrane after $\mathrm{CO}_{2}$ treatment (supplemental Fig. $1 \mathrm{~A}$, available at www. jneurosci.org as supplemental material), suggesting that the change in Kv2.1 localization in the brains of $\mathrm{CO}_{2}$-treated animals was attributable to a lateral dispersion in the plasma membrane. $\mathrm{CO}_{2}$ anesthesia also led to disruption of the unusually large (2-5 $\mu$ m diameter) Kv2.1 clusters (supplemental Fig. $1 B$, available at www.jneurosci.org as supplemental material) associated with muscarinic synapses in motor neurons (Muennich and Fyffe, 2003). These results demonstrate that brief $\mathrm{CO}_{2}$ inhalation in both conscious and unconscious rats rapidly changes the phosphorylation state and localization of Kv2.1 in vivo and, in some cells, their association with specialized synapses. As such, previous results obtained from $\mathrm{CO}_{2}$-anesthesized animals may have 


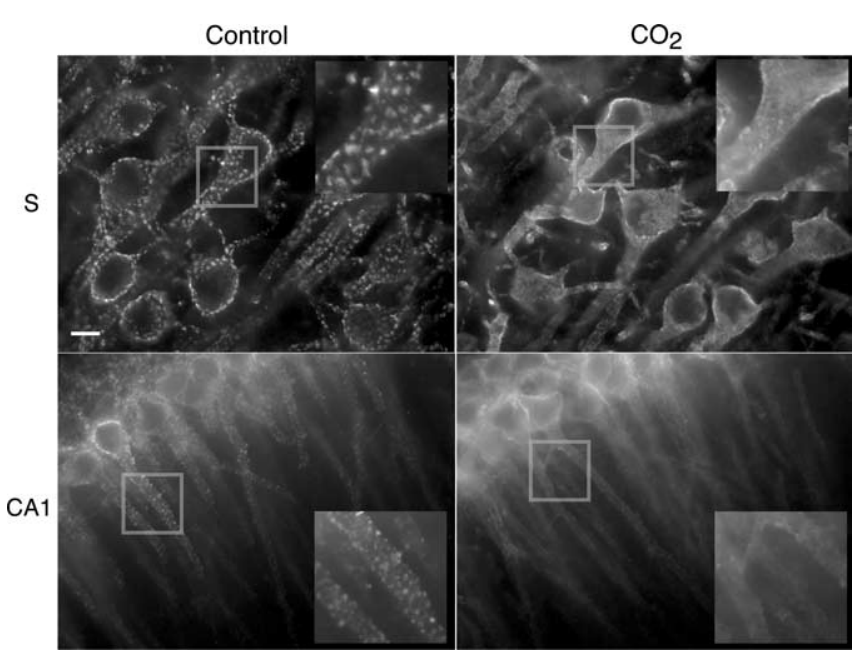

Figure 2. $\mathrm{CO}_{2}$ dramatically changes localization of Kv2.1 in vivo. Rats were intraperitoneally injected with $50 \mathrm{mg} / \mathrm{kg}$ pentobarbital and exposed to air (control) or $\mathrm{CO}_{2}$ for $2 \mathrm{~min}$ in a closed chamber. Brain sections from these control and $\mathrm{CO}_{2}$-treated rats were stained with anti-Kv2.1 antibody. Images were taken by conventional fluorescence microscopy from the subiculum ( $S$ ) and CA1 region of the hippocampus. Insets are higher-magnification views of Kv2.1 staining corresponding to the boxed areas in the main images. Scale bar, $10 \mu \mathrm{m}$.

yielded an underestimation of the full extent of Kv2.1 phosphorylation and clustering in brain neurons. Moreover, these dynamic changes in Kv2.1 localization in vivo could impact its role in regulating somatic and dendritic excitability.

\section{Ischemia induces Kv2.1 dephosphorylation in culture and in vivo}

To begin to investigate the neuronal signaling mechanisms underlying the dramatic effects of $\mathrm{CO}_{2}$ inhalation in vivo, we examined the effects of $\mathrm{CO}_{2}$ exposure and acidified culture medium, mimicking tissue acidosis, on Kv2.1 in cultured hippocampal neurons. We found that the phosphorylation state of Kv2.1 was not altered during exposure of neurons to $\mathrm{pH} 6.6$ medium or elevated $\mathrm{CO}_{2}$ for $20 \mathrm{~min}$ (Fig. $3 \mathrm{~A}$ ) mixed in ambient air to avoid excessive anoxia. However, inhibition of oxidative ATP synthesis, such as occurs in conditions of hypoxia/ischemia, by exposing cultured neurons to CI (5 mm sodium azide and $5 \mathrm{~mm}$ 2-deoxyD-glucose) (Swanson and Benington, 1996) for $10 \mathrm{~min}$ induced Kv2.1 dephosphorylation (Fig. 3B). The intracellular ATP levels in cultured neurons were decreased to $17.1 \pm 2.0 \%$ of control levels by $5 \mathrm{~min}$ of CI and to $8.8 \pm 2.0 \%$ by $15 \mathrm{~min}$ of CI, consistent with a previous report (Bernstein and Bamburg, 2003). Although blockade of voltage-dependent $\mathrm{Na}^{+}$channels with TTX partially but not significantly ( $p=0.08)$ inhibited dephosphorylation of Kv2.1 induced by $10 \mathrm{~min}$ of CI (Fig. 3B), CI for 15 min even in the presence of $1 \mu \mathrm{M}$ TTX yielded full induction of Kv2.1 dephosphorylation (Fig. 3C), suggesting that the CI effects occur essentially independent of membrane depolarization. The CI-induced Kv2.1 dephosphorylation was completely reversible during CI washout (supplemental Fig. $2 \mathrm{~A}$, available at www.jneurosci.org as supplemental material). Thus, as shown previously for glutamate-induced Kv2.1 dephosphorylation (Misonou et al., 2004), neurons can reestablish the normal Kv2.1 phosphorylation state after this brief ischemic insult.

The efficacy of CI but not media acidosis nor $\mathrm{CO}_{2}$ itself on Kv2.1 dephosphorylation in cultured neurons led us to reinvestigate the basis for $\mathrm{CO}_{2}$-induced Kv2.1 dephosphorylation in vivo. Brains from pentobarbital-anesthetized rats were subjected to complete global ischemia for $4 \mathrm{~min}$ after decapitation (Namba et al., 2002), which induced Kv2.1 dephosphorylation in vivo (Fig. 3D). Overdose itself with pentobarbital ( $150 \mathrm{mg} / \mathrm{kg})$ over a 4 min period did not elicit Kv2.1 dephosphorylation (Fig. 3D), indicating that Kv2.1 dephosphorylation is not rapid postmortem changes. Together, these results suggest that the $\mathrm{CO}_{2}$ effects in vivo are primarily attributable to hypoxia but not hypercapnia and that metabolic inhibition by hypoxia/ischemia in vivo and by $\mathrm{CI}$ in vitro induces rapid dephosphorylation of Kv2.1.

\section{Chemical ischemia modulates the phosphorylation state and localization of Kv2.1 through calcineurin activation}

We next began to investigate the signaling pathway underlying the CI-induced dephosphorylation of Kv2.1. We found that, unlike the effects of glutamate (Misonou et al., 2004), the CI effects occurred in the absence of extracellular $\mathrm{Ca}^{2+}$ (Fig. 4A). Antagonists for ionotropic glutamate receptors (GluRs) (AP-5 and CNQX; GluR blockers) that predictably blocked the effects of glutamate (Misonou et al., 2004) did not affect induction by CI (Fig. $4 B$ ), showing that the CI effects were not mediated by secondary and incidental glutamate release. This conclusion was further supported by the result that blockade of exocytosis by botulinum neurotoxin type B also did not affect CI-induced Kv2.1 dephosphorylation, although the toxin effectively reduced the levels of vesicle-associated membrane protein-2 (data not shown).

The effects of glutamate on Kv2.1 phosphorylation in cultured hippocampal neurons are mediated by calcineurin (Misonou et al., 2004). Interestingly, the CI-induced effects on Kv2.1 dephosphorylation were also significantly $(p=0.003)$ blocked by the calcineurin inhibitors cyclosporin A (Fig. 4C) and FK520 (5 $\mu \mathrm{M})$, a more widely available FK506 analog (supplemental Fig. $2 B$, available at www.jneurosci.org as supplemental material). These results suggest a CI-induced signaling pathway distinct in its upstream events from that induced by glutamate but similar in its convergence on calcineurin-dependent Kv2.1 dephosphorylation.

Given that $\mathrm{CO}_{2}$ inhalation in vivo and glutamate stimulation in vitro (Misonou et al., 2004) lead to both Kv2.1 dephosphorylation and dispersion of Kv2.1 clusters, we next examined the effects of CI on Kv2.1 localization in cultured neurons. Cultured hippocampal pyramidal neurons have distinct Kv2.1 clusters on their somatodendritic membrane (Fig. 4D) similar to those seen on pyramidal neurons in vivo (Fig. 2). CI caused dispersion of somatodendritic Kv2.1 clusters in cultured neurons (Fig. 4E), similar to the effects of glutamate stimulation (Misonou et al., 2004). As such, the majority of neurons treated with CI $(74.7 \pm 3.6 \%)$ showed diffuse somatodendritic Kv2.1 staining compared with a small minority of cells $(13.3 \pm 1.7 \%)$ in control cultures. Note that these cultured neurons have an additional pool of Kv2.1 on the axon initial segment, a culture artifact (Lim et al., 2000) not seen in brain neurons (Fig. 2), and this pool of Kv2.1 was not affected by glutamate or CI treatment. Importantly, the effects of CI on Kv2.1 localization were significantly inhibited by cyclosporin A $(31.4 \pm 2.2 \%$ cells showed a diffuse Kv2.1 pattern; $p<0.001$ compared with CI-treated cells in the absence of cyclosporin A) (Fig. $4 F$ ), supporting a role for calcineurin in the CI-induced effects on Kv2.1 phosphorylation and localization.

\section{Chemical ischemia increases $\left[\mathrm{Ca}^{2+}\right]_{\mathrm{i}}$ by inducing $\mathrm{Ca}^{2+}$} release from intracellular stores

Given the lack of a requirement for extracellular $\mathrm{Ca}^{2+}$ in the CI-induced effects on Kv2.1 and that increased $\left[\mathrm{Ca}^{2+}\right]_{\mathrm{i}}$ is neces- 
sary and sufficient to induce the calcineurin-dependent dephosphorylation of Kv2.1 during glutamate stimulation (Misonou et al., 2004), we next investigated whether the CI effects were mediated by $\mathrm{Ca}^{2+}$ release from intracellular stores. $\mathrm{Ca}^{2+}$ imaging of cultured hippocampal neurons was performed using Fluo-4 and confocal laser scanning microscopy, focusing on changes in Fluo-4 fluorescence intensity on the soma and proximal dendrites in which Kv2.1 is localized. Hippocampal neurons exhibited spontaneous $\left[\mathrm{Ca}^{2+}\right]_{\mathrm{i}}$ transients whose frequency and amplitude were suppressed by $1 \mu \mathrm{M}$ tetrodotoxin (data not shown). CI was then induced, leading to a gradual increase in Fluo-4 signal intensity over $5 \mathrm{~min}$ (Fig. $5 A, B$ ), as reported previously (Chow and Haddad, 1998). As shown in Figure 5B, CI induced a large and sustained increase in $\left[\mathrm{Ca}^{2+}\right]_{\mathrm{i}}$ in the absence of extracellular $\mathrm{Ca}^{2+}$. This CI-induced increase in $\left[\mathrm{Ca}^{2+}\right]_{\mathrm{i}}$ exhibited slower kinetics than that induced by glutamate stimulation in the presence of extracellular $\mathrm{Ca}^{2+}$, as reported for cultured cortical neurons (Chow and Haddad, 1998). However, the CI-induced increases in $\left[\mathrm{Ca}^{2+}\right]_{\mathrm{i}}$ reached levels comparable with those seen during glutamate stimulation within 5 min of CI induction (Fig. $5 B$ ).

To pharmacologically investigate the source of intracellular $\mathrm{Ca}^{2+}$ release, we examined the effects of thapsigargin, an inhibitor of the sarcoplasmic/endoplasmic reticulum (ER) $\mathrm{Ca}^{2+}$-ATPase caffeine to induce $\mathrm{Ca}^{2+}$ release from ryanodine-sensitive $\mathrm{Ca}^{2+}$ stores, and carbonyl cyanide $p$-trifluoromethoxyphenylhydrazone (FCCP), which induces $\mathrm{Ca}^{2+}$ release from mitochondria by eliminating the $\mathrm{H}^{+}$gradient (Yang et al., 2003). Neither thapsigargin nor caffeine induced significant Kv2.1 dephosphorylation (data not shown). However, treatment with FCCP alone resulted in an increase in $\left[\mathrm{Ca}^{2+}\right]_{\mathrm{i}}$ similar to that obtained with CI (Fig. 5C), as well as dephosphorylation of Kv2.1 (supplemental Fig. 2C, available at www. jneurosci.org as supplemental material) in the absence of extracellular $\mathrm{Ca}^{2+}$. Importantly, FCCP treatment after induction of CI yielded no additional increases in $\left[\mathrm{Ca}^{2+}\right]_{\mathrm{i}}$ (Fig. $5 \mathrm{C}$ ), suggesting that the CI- and FCCP-induced $\mathrm{Ca}^{2+}$ release was from the same intracellular pool (i.e., mitochondria). Together, these data suggest that $\mathrm{CI}$ induces $\mathrm{Ca}^{2+}$ release from intracellular stores, presumably from mitochondria, and that the resultant increase in $\left[\mathrm{Ca}^{2+}\right]_{i}$ is sufficient to induce calcineurindependent dephosphorylation of Kv2.1. antibody (red).
A

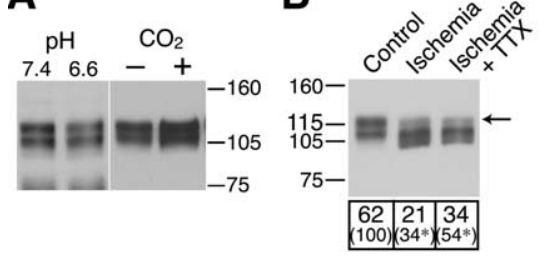

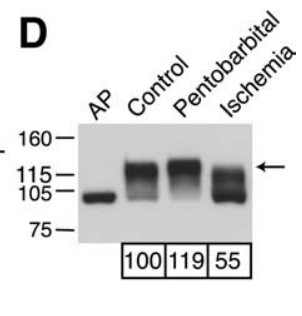

Figure 3. Ischemia induces dephosphorylation of Kv2.1 in vitro and in vivo. A, Cultured hippocampal neurons were incubated in buffer at pH 7.4 or 6.6 or treated in a chamber filled with $\mathrm{CO}_{2}$ gas at $37^{\circ} \mathrm{C}$ for $20 \mathrm{~min}$. Proteins were solubilized in SDS sample buffer, fractionated on SDS-PAGE, and analyzed for Kv2.1 by immunoblotting. $\boldsymbol{B}$, Neurons were incubated with $\mathrm{Cl}$ reagents $(5 \mathrm{~mm}$ 2-deoxy-D-glucose and $5 \mathrm{~mm}$ sodium azide; Ischemia) in the presence or absence of $1 \mu \mathrm{m}$ TTX for $10 \mathrm{~min}$. Bands of total and phosphorylated ( $M_{r}$ of $125 \mathrm{kDa}$, indicated by an arrow) Kv2.1 were quantified. Values in boxes indicate the amount of phosphorylated Kv2.1 ( $\mathrm{M}_{\mathrm{r}}$ of $125 \mathrm{kDa}$ ) as a percentage of total Kv2.1 and are the mean values from five independent experiments (control, $62.0 \pm 4.7 \%$; ischemia, $21.0 \pm 4.5 \%$; ischemia plus TTX, 33.5 \pm 0.5\%). Values in parentheses denote the level of phosphorylated Kv2.1 shown as a percentage of the control level. Statistical significance between the control and experimental values are shown by asterisks ( $p<0.01, n=5$ ). C, Neurons were incubated with Cl reagents (Chemical ischemia) for 2, 5, 10, or 15 min in the presence of $1 \mu \mathrm{m}$ tetrodotoxin. The levels of phosphorylated Kv2.1 were as follows: control, $100.0 \pm 7.6 \%$; $15 \mathrm{~min}$ of $\mathrm{Cl}, 23.5 \pm$ 3.1\%. ${ }^{*} p<0.01(n=3)$. D, Animals were decapitated (Control) or injected with $150 \mathrm{mg} / \mathrm{kg}$ pentobarbital. To induce complete global ischemia, decapitated heads were incubated for $4 \mathrm{~min}$ at room temperature (Ischemia). Crude brain membranes were prepared from these animals, and proteins were analyzed by immunoblotting for Kv2.1. A fraction of crude membranes was incubated with $0.1 \mathrm{U} / \mathrm{ml} \mathrm{AP.} \mathrm{The} \mathrm{levels} \mathrm{of} \mathrm{phosphorylated} \mathrm{Kv2.1} \mathrm{were} \mathrm{as} \mathrm{follows:} \mathrm{control,} 100.0 \pm 3.3 \%$; global ischemia, $55.4 \pm$ $0.7 \%$. Numbers to left or right denote mobility of molecular weight standards in kilodaltons.
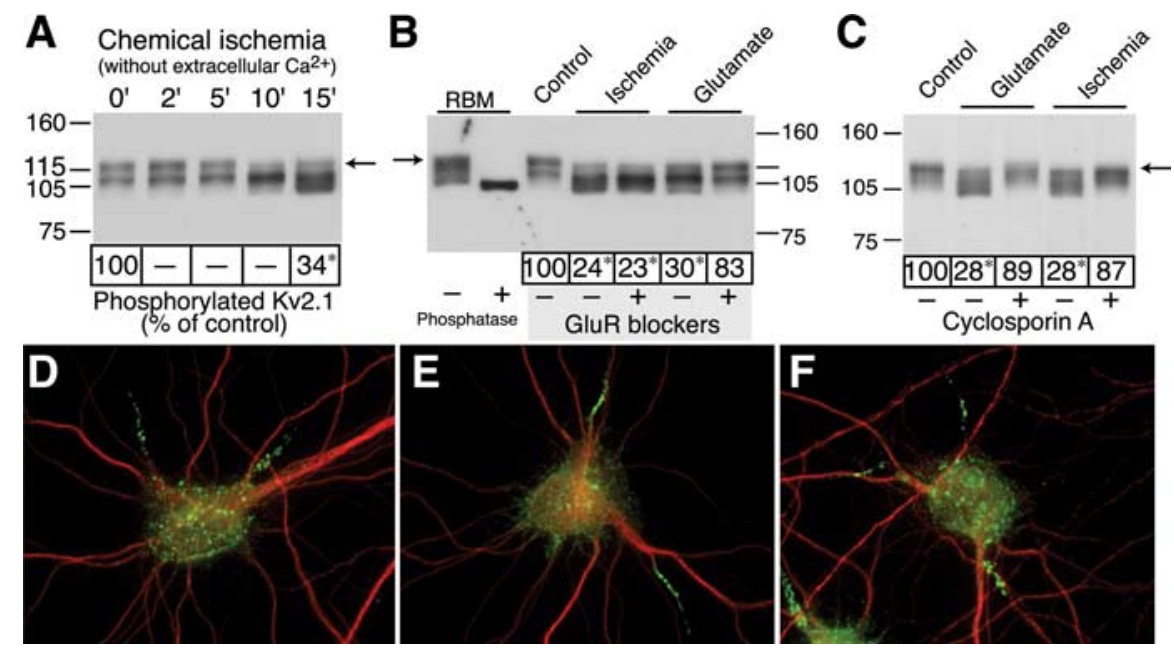

Figure 4. Chemical ischemia changes Kv2.1 phosphorylation state and localization by driving $\mathrm{Ca}^{2+}$ from the intracellular stores and activating calcineurin. $\boldsymbol{A}$, Neurons were incubated with Cl reagents for 2,5,10, and $15 \mathrm{~min}$ in the absence of extracellular $\mathrm{Ca}^{2+}$. Values in boxes indicate the level of phosphorylated Kv2.1 (marked by an arrow) shown as a percentage of the control level (control, $100 \pm 6.4 \%$; $15 \mathrm{~min}$ of $\mathrm{Cl}, 34.1 \pm 7.2 \%)(n=3)$. $\boldsymbol{B}$, Neurons were incubated with $20 \mu \mathrm{m}$ glutamate or $\mathrm{Cl}$ reagents (Ischemia) for $15 \mathrm{~min}$, in the presence or absence of $100 \mu \mathrm{M} \mathrm{AP-5}$ and $10 \mu \mathrm{M}$ CNQX (GluR blockers) in the presence of $1 \mu \mathrm{M}$ tetrodotoxin. The levels of phosphorylated Kv2.1 were as follows: control, $100 \pm 6.8 \%$; ischemia, $24.2 \pm 2.2 \%$; ischemia plus GluR blockers, $22.7 \pm 2.4 \%$; glutamate, $30.4 \pm 4.6 \%$; glutamate plus GluR blockers, $83.4 \pm 1.7 \%(n=3)$. RBM, Rat brain membrane fraction. C, Neurons were incubated with $20 \mu \mathrm{m}$ glutamate or $\mathrm{Cl}$ reagents for $15 \mathrm{~min}$, in the presence or absence of 20 $\mu \mathrm{m}$ cyclosporin A in the presence of $1 \mu \mathrm{m}$ tetrodotoxin. The levels of phosphorylated Kv2.1 were as follows: control, $100 \pm 6.8 \%$; glutamate, $28.4 \pm 2.8 \%$; glutamate plus cyclosporin A, $89.1 \pm 5.5 \%$; ischemia, $28.2 \pm 5.4 \%$; ischemia plus cyclosporin $A$, $86.5 \pm 9.7 \%(n=3)$. Proteins were solubilized in SDS sample buffer, fractionated on SDS-PAGE, and analyzed for Kv2.1 by immunoblotting. Statistical significance between control and experimental values $(\boldsymbol{A}, \boldsymbol{B})$ or between values in the presence and absence of cyclosporin A ( $C$ are shown by asterisks $(p<0.01)$. Numbers to left or right denote mobility of molecular weight standards in kilodaltons. D-F, Neurons were incubated without $(\boldsymbol{D})$ or with $(\boldsymbol{E})$ Cl reagents, or Cl reagents and $20 \mu \mathrm{m}$ cyclosporin A $(\boldsymbol{F})$ for $15 \mathrm{~min}$. Cells were fixed with 4\% paraformaldehyde and stained with anti-Kv2.1 antibody (green) and anti-MAP-2

Chemical ischemia alters the voltage-dependent activation of neuronal $I_{\mathrm{K}}$ and neuronal activity

Whole-cell patch-clamp recording from cultured hippocampal neurons revealed that $\mathrm{CI}$ led to large hyperpolarizing shifts (control, $V_{1 / 2}$ of $+16.6 \pm 0.4 \mathrm{mV} ; \mathrm{CI}, V_{1 / 2}$ of $-4.3 \pm 0.4 \mathrm{mV} ; p<$ 0.001 vs control; $n=5$ ) in the voltage dependence of activation of 

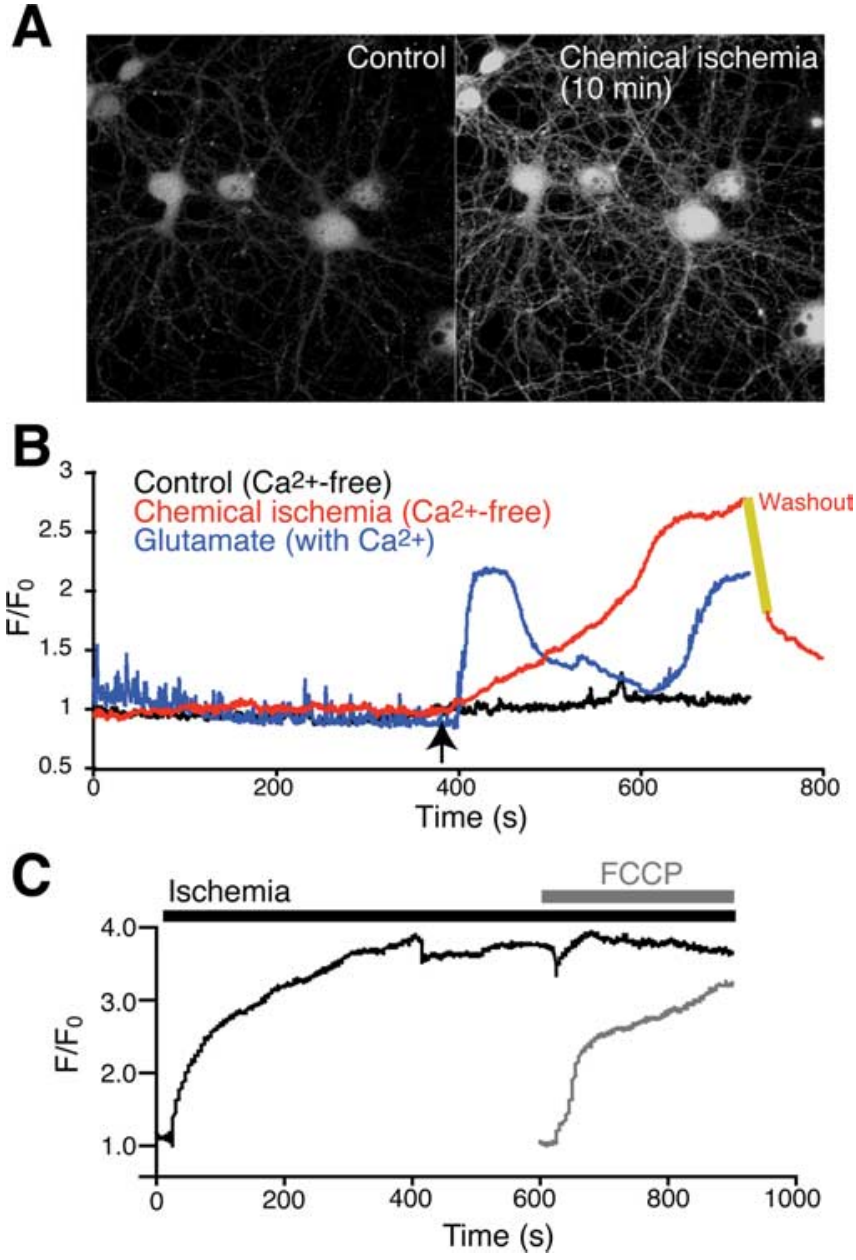

Figure 5. Chemical ischemia induces $\mathrm{Ca}^{2+}$ release from intracellular stores. $\boldsymbol{A}$, Neurons were loaded with $5 \mu \mathrm{m}$ Fluo-4 and incubated with Cl reagents ( $5 \mathrm{~mm}$ 2-deoxy-o-glucose and 5 mm sodium azide) for $10 \mathrm{~min}$ in the presence of $1 \mu \mathrm{m}$ tetrodotoxin. $\boldsymbol{B}$, (l-induced increase of $\left[\mathrm{Ca}^{2+}\right]_{\mathrm{i}}$ in the absence of extracellular $\mathrm{Ca}^{2+}$. Cl reagents or glutamate $(10 \mu \mathrm{m})$ were added at the point indicated by an arrow. Neurons were incubated with glutamate in the presence of extracellular $\mathrm{Ca}^{2+}$. Thick yellow line represents the absence of recording during the washout period. $C$, Role of mitochondria as a source of released $\mathrm{Ca}^{2+}$. Neurons were incubated without (gray trace) or with (black trace) CI reagents for $10 \mathrm{~min}$ and then incubated with $1 \mu \mathrm{m} \mathrm{FCCP} \mathrm{for}$ $5 \mathrm{~min}$. Imaging rate, $1 \mathrm{~Hz}$.

$I_{\mathrm{K}}$, without any significant change in the slope of the activation curve (Fig. 6A,B). These CI-induced changes are comparable with those induced by glutamate stimulation (Misonou et al., 2004). As a result of the CI-induced change in voltage-dependent activation, increased $I_{\mathrm{K}}$ amplitude was observed at each test potential (Fig. 6A,B). These CI-induced shifts in voltage-dependent activation of $I_{\mathrm{K}}$ were blocked by pretreatment with FK520 ( $V_{1 / 2}$ of $+13.1 \pm 0.5 \mathrm{mV}$ ) (Fig. $6 B$ ), suggesting that the calcineurindependent dephosphorylation of Kv2.1 was responsible for the altered biophysical properties of $I_{\mathrm{K}}$. Previous studies have shown that the bulk ( $\geq 80 \%)$ of $I_{\mathrm{K}}$ in hippocampal and cortical pyramidal neurons is contributed by Kv2.1 (Murakoshi and Trimmer, 1999; Du et al., 2000; Pal et al., 2003). We found that the broadspectrum $\mathrm{K}^{+}$channel blocker TEA when used at $5 \mathrm{~mm}$ [the $\mathrm{K}_{\mathrm{i}}$ for TEA block of Kv2.1 is $1-5 \mathrm{~mm}$ based on studies of recombinant rat brain Kv2.1 in heterologous cells (Shi et al., 1994; Immke et al., 1999)] effectively blocked ( $90.0 \pm 0.2 \%$ inhibition) $I_{\mathrm{K}}$ in control neurons. These data suggest that TEA effectively blocks the major $\mathrm{Kv} 2.1$ component of $I_{\mathrm{K}}$. Interestingly, a similar level of TEA inhibition was obtained for the increased $I_{\mathrm{K}}$ in neurons after induc-
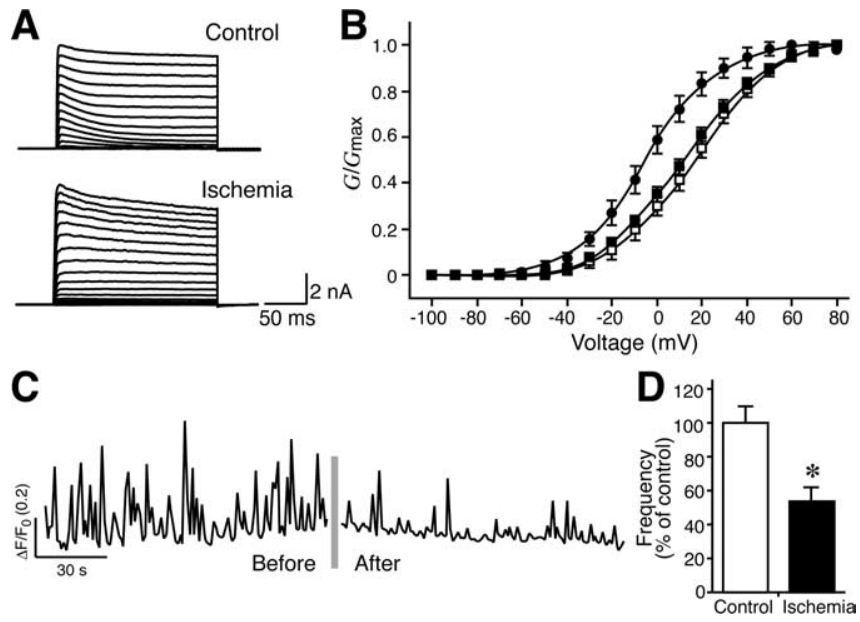

Figure 6. Chemical ischemia alters the properties of $I_{K}$ current and suppresses neuronal excitability. $\boldsymbol{A}$, Representative $I_{\mathrm{K}}$ currents in a cultured hippocampal neuron recorded under whole-cell voltage clamp. The membrane potential was held at $-100 \mathrm{mV}$ and depolarized from the holding potential of $-100 \mathrm{mV}$ to voltages between -90 and $+80 \mathrm{mV}$ in $10 \mathrm{mV}$ increments for $200 \mathrm{~ms}$. A $30 \mathrm{~ms}$ prepulse to $-10 \mathrm{mV}$ was given before each test depolarization to eliminate transient $\mathrm{K}^{+}$current. $\boldsymbol{B}$, The plot shows the conductance-voltage $(G-V)$ relationship of peak $I_{K}$ currents recorded from neurons before (open square), after (filled circle) $\mathrm{Cl}$, and after $\mathrm{Cl}$ in the presence of $5 \mu \mathrm{m}$ FK520 (filled square). C, Suppression of spontaneous $\mathrm{Ca}^{2+}$ transients after $\mathrm{Cl}$. Neurons were loaded with $5 \mu \mathrm{m}$ Fluo-4. Representative spontaneous $\mathrm{Ca}^{2+}$ bursts in the soma before and after $\mathrm{Cl}$. Imaging rate, $1 \mathrm{~Hz}$. D. Spontaneous $\mathrm{Ca}^{2+}$ bursts with $\Delta F / F_{0}>0.2$ in the soma for $2 \mathrm{~min}$ were analyzed in cells before (Control) and after (Ischemia) the treatment with Cl reagents. ${ }^{*} p<0.01(n=8)$.

tion of $\mathrm{CI}(92.0 \pm 1.4 \%$ inhibition), suggesting that the bulk of the enhanced $I_{\mathrm{K}}$ in ischemic neurons can be attributed to Kv2.1. A mixture of iberiotoxin (IbTx, $10 \mathrm{nM}$ ), a specific blocker of largeconductance $\mathrm{Ca}^{2+}$-activated $\mathrm{BK} \mathrm{K}{ }^{+}$channels, and apamin $(500$ $\mathrm{nM})$, a specific blocker of small-conductance $\mathrm{Ca}^{2+}$-activated SK channels, yielded minimal block (12.0 $\pm 13.3 \%$ inhibition $)$ of the total outward $\mathrm{K}^{+}$current in these neurons.

Because such large changes in the activation properties of neuronal $I_{\mathrm{K}}$ would be predicted to dramatically alter neuronal excitability (Surmeier and Foehring, 2004), we next examined whether CI changes the electrical activity of neurons, using spontaneous $\left[\mathrm{Ca}^{2+}\right]_{\mathrm{i}}$ transients as an indirect readout of activity. As shown in Figure 6C, control hippocampal neurons exhibited regular spontaneous $\left[\mathrm{Ca}^{2+}\right]_{\mathrm{i}}$ transients. Neurons subjected to $\mathrm{CI}$ (10 $\min )$ and washout $\left(10 \mathrm{~min}\right.$, which restores resting $\left.\left[\mathrm{Ca}^{2+}\right]_{\mathrm{i}}\right)($ Fig. $5 B)$ showed a significantly $(\approx 50 \% ; p=0.005$ compared with control) depressed frequency of spontaneous $\left[\mathrm{Ca}^{2+}\right]_{\mathrm{i}}$ transients of $\Delta F / F_{0}>0.2$ (Fig. $6 C, D$ ). The overall $\mathrm{Ca}^{2+}$ load (obtained by integrating changes in spontaneous $\left[\mathrm{Ca}^{2+}\right]_{\mathrm{i}}$ transients over a 2 min period) was also significantly reduced (supplemental Fig. $3 \mathrm{~A}$, available at www.jneurosci.org as supplemental material). Furthermore, FK520 pretreatment reversed this suppression $(p=$ 0.04 ) (supplemental Fig. $3 A$, available at www.jneurosci.org as supplemental material), indicating that the postischemic suppression of neuronal excitability results from calcineurindependent events.

\section{A neuroprotective mechanism conferred by TEA-sensitive} and iberiotoxin-insensitive $\mathrm{K}^{+}$channels

To further understand the role of $I_{\mathrm{K}} / \mathrm{Kv} 2.1$ modulation in the suppression of neuronal activity, we examined whether TEA could reverse the suppression of neuronal excitability induced by CI. As shown in Figure 7, neurons incubated with $5 \mathrm{~mm}$ TEA (which blocks $>90 \%$ of $I_{\mathrm{K}}$ in these cultured hippocampal neu- 
rons; see above) showed initial burst activity followed by recovery of relatively normal spontaneous $\mathrm{Ca}^{2+}$ transients. However, TEA treatment of neurons that had been subjected to CI for 10 min elicited $\mathrm{Ca}^{2+}$ overload (Fig. 7) (supplemental Fig. $3 B, C$, available at www.jneurosci.org as supplemental material). In contrast to the dramatic effects of TEA, $10 \mathrm{~nm} \mathrm{IbTx}$ did not have any noticeable effect on CIinduced $\left[\mathrm{Ca}^{2+}\right]_{\mathrm{i}}$, at least within the $4 \mathrm{~min}$ window of imaging (data not shown). These results show that, after brief ischemic insults, neurons exhibit hyperexcitability that can be suppressed by TEAsensitive IbTx-insensitive $I_{\mathrm{K}}$ currents.

To examine whether $I_{\mathrm{K}}$-mediated suppression of excitability would affect neuronal viability after brief ischemic insults, we assessed the neuronal damage induced by $\mathrm{CI}$ in the presence of TEA. Neurons were subjected to CI for $10 \mathrm{~min}$, washed, and then incubated with or without TEA for $1 \mathrm{~h}$. Cell viability was quantified after $24 \mathrm{~h}$ incubation under normal culture conditions. As shown in Figure 8, brief CI in itself did not affect neuronal viability, suggesting that neurons have protective mechanisms against this extent of ischemic insult. However, brief exposure of cells to $5 \mathrm{~mm}$ TEA just after CI resulted in significant neuronal damage, whereas TEA treatment in itself had little effect on cell viability (Fig. $8 \mathrm{~B})$. IbTx (10 nM) did not affect neuronal viability in the presence (Fig. $8 \mathrm{~B}$ ) or absence (data not shown) of ischemic insult. These results suggest that TEA-sensitive and IbTx-insensitive $I_{\mathrm{K}}$ channels, of which a large component is $\mathrm{Kv} 2.1$, confer protection against ischemic neuronal damage by suppressing neuronal hyperexcitability.

Together, these results suggest that hypoxia/ischemia in vivo and in vitro induces calcineurin-mediated Kv2.1 dephosphorylation that leads to dramatic changes in its localization and function. Such modulation of Kv2.1 represents a novel protective mechanism activated in response to brief ischemia insults.

\section{Discussion}

The major findings of this study are that brief ischemic insults in vivo and in vitro induce dephosphorylation of Kv2.1, loss of Kv2.1 clustering, and a large hyperpolarizing shift in the voltagedependent activation of $\mathrm{Kv} 2.1 / I_{\mathrm{K}}$ channels. The ischemiainduced modulation of Kv2.1 is distinct from that induced in central neurons by seizures in vivo and by glutamate stimulation in vitro (Misonou et al., 2004) in that it occurs in the absence of extracellular $\mathrm{Ca}^{2+}$ and is not dependent on ischemia-induced seizures in vivo or glutamate release in vitro (supplemental Fig. 4, available at www.jneurosci.org as supplemental material). The effects of ischemia in vivo are also more widespread and are observed throughout the brain, whereas the effects induced by kainate-induced seizures are restricted to the limbic system (Misonou et al., 2004). Despite these distinctions, increased $\left[\mathrm{Ca}^{2+}\right]_{\mathrm{i}}$ and calcineurin remain central to both responses. Moreover, both the glutamate- and ischemia-induced effects on Kv2.1 are reversible, in that removal of either stimulus leads to recovery of phosphorylated Kv2.1 and Kv2.1 surface clusters and restoration of the relatively depolarized voltage-dependent activation typical of control neurons. Although release of $\mathrm{Ca}^{2+}$ from intracellular stores, specifically mitochondria as opposed to glutamate- stimulated $\mathrm{Ca}^{2+}$ influx via ionotropic glutamate receptors, seems to be critical to the ischemia-induced modulation of Kv2.1, sustained increases in $\left[\mathrm{Ca}^{2+}\right]_{\mathrm{i}}$ leading to activation of calcineurin remain key. As predicted for the effects of glutamate stimulation (Surmeier and Foehring, 2004; Misonou et al., 2005), brief ischemic insult and enhanced activation of Kv2.1 leads to a suppression of neuronal activity (supplemental Fig. 4, available at www. jneurosci.org as supplemental material). The $\mathrm{K}^{+}$channel blocker TEA eliminates CI-induced suppression of neuronal excitability, and TEA treatment after brief and otherwise nonlethal exposure to CI leads to cell death. These findings show that neurons can use different upstream signaling pathways to achieve reversible calcineurin-dependent regulation of Kv2.1 localization and function in response to both hyperexcitability and ischemia. These studies also provide evidence that the activity of Kv2.1, although classically defined as a strictly voltage-dependent delayed rectifier $\mathrm{K}^{+}$channel, is also dependent on metabolic state and $\left[\mathrm{Ca}^{2+}\right]_{\mathrm{i}}$.

\section{Potential roles for Kv2.1 modulation in brain ischemia}

Neurons need protection against excessive hyperactivity, which could lead to pathological excitotoxic conditions. It is widely accepted that neurons have developed a diverse array of immediate and long-term homeostatic protective mechanisms that respond to abnormal hyperactivity. Hypoxia and ischemia cause membrane depolarization, and the generalized seizures that can be evoked by such metabolic stress can aggravate brain damage (Calabresi et al., 2003). However, different neurons have distinct immediate and long-term protective mechanisms "on demand" to suppress the hyperexcitability induced by brief hypoxia/ischemia. One immediate mechanism is the depression of neuronal excitability during hypoxia/ischemia-induced activation of neuronal $\mathrm{K}_{\mathrm{ATP}}$ channels (Ballanyi, 2004), especially in neurons in anoxia-tolerant brain regions such as substantia nigra pars reticulata (Yamada et al., 2001), dorsal vagal neurons, and cerebellar Purkinje cells (Ballanyi, 2004). However, many brain neurons (e.g., hippocampal pyramidal neurons) express lower levels of functional $\mathrm{K}_{\mathrm{ATP}}$ channels (Zawar and Neumcke, 2000; Griesemer et al., 2002), such that brief hypoxia and ischemia in hippocampal slices induces immediate seizure-like hyperactivity followed by a delayed and prolonged suppression of neuronal activity (Kawasaki et al., 1990; Yamamoto et al., 1997). In cultured 
A
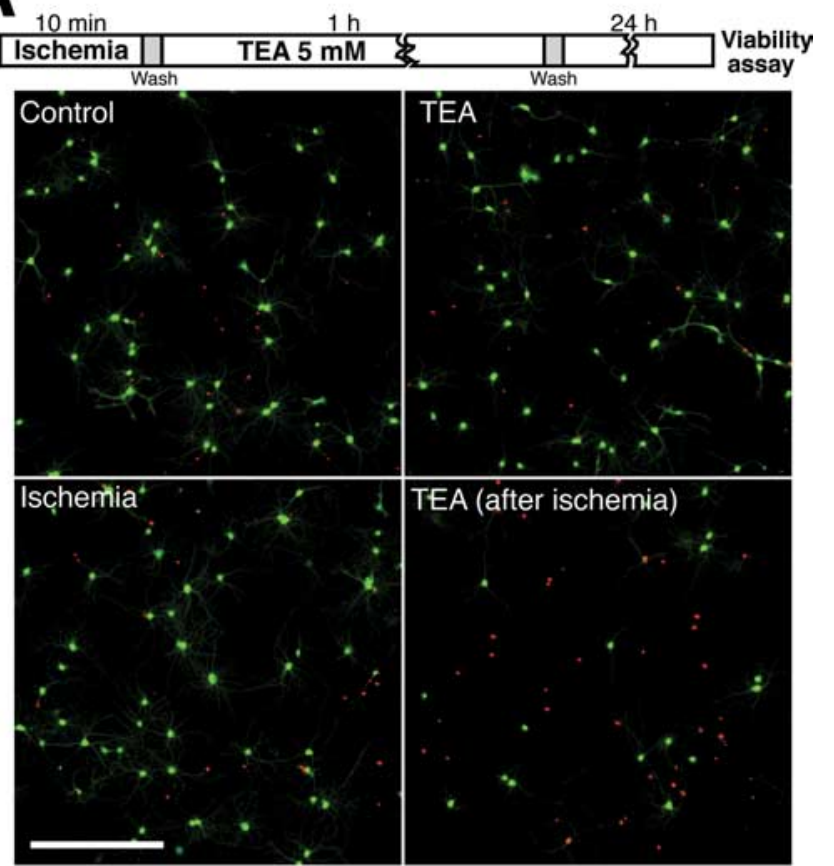

B

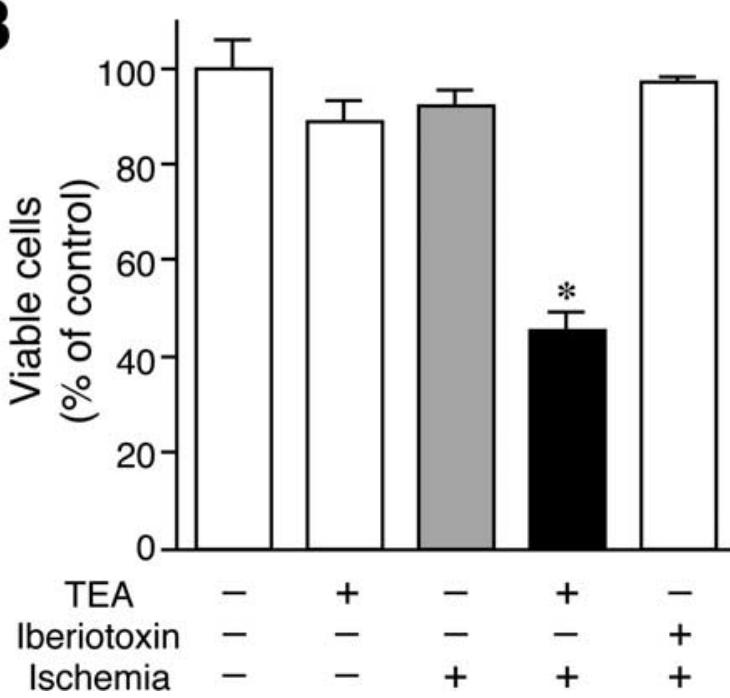

Figure 8. Tetraethylammonium aggravates neuronal damage after ischemic insults. $\boldsymbol{A}, \mathrm{Neu}$ rons were incubated with Cl reagents ( $5 \mathrm{~mm} 2$-deoxy-D-glucose and $5 \mathrm{~mm}$ sodium azide) for 10 min, washed, and then incubated with $5 \mathrm{~mm}$ TEA for $1 \mathrm{~h}$. After the incubation, cells were kept in the normal culture condition for $24 \mathrm{~h}$ and subjected to the cell viability assay as illustrated in the top schematic. All treatments were done in the absence of tetrodotoxin. Representative images taken with a $2.5 \times$ objective were shown in the bottom panels. Scale bar, $500 \mu \mathrm{m}$. B, The number of total and viable cells were counted (total of $>200$ cells were counted in each sample) and shown as percentages of viable cells in control. Iberiotoxin (10 nм) was added as described for TEA. Data are the means $\pm \operatorname{SEM}(n=5) .{ }^{*} p<0.01$

hippocampal neurons, the suppressed neuronal activity in response to hypoxia/ischemia is attributable to an increase in overall $\mathrm{K}^{+}$current. Previous studies have attributed this increase to large-conductance $\mathrm{Ca}^{2+}$-activated $\mathrm{BK}$ channels, based on the fact that the increases in $\mathrm{K}^{+}$current are dependent on increased $\left[\mathrm{Ca}^{2+}\right]_{\mathrm{i}}$ (Yamamoto et al., 1997; Erdemli et al., 1998; Tanabe et al., 1999), although more recent studies used more definitive pharmacological blockade (Runden-Pran et al., 2002). That ischemia also modulates $\mathrm{Kv} 2.1$ in a $\mathrm{Ca}^{2+}$-dependent manner, through the activation of the protein phosphatase calcineurin, suggests that $\mathrm{Kv} 2.1 / I_{\mathrm{K}}$ may also contribute to ischemia-induced increases in neuronal $\mathrm{K}^{+}$current. Moreover, the bulk of wholecell $I_{\mathrm{K}}$ in hippocampal neurons under both control and CI conditions, as well as the CI-induced suppression of neuronal excitability, are blocked by TEA doses that yield effective blockade of Kv2.1 and BK channels but not by the specific BK channel blocker IbTx. Previous studies showed that knockdown of Kv2.1/I $I_{\mathrm{K}}$ in cultured hippocampal slices by antisense oligonucleotides leads to neuronal hyperexcitability, but only under periods of highfrequency stimulation (Du et al., 2000). Conversely, when $\mathrm{Kv} 2.1 / I_{\mathrm{K}}$ is potentiated by arachidonic acid, neurons are protected from 4-aminopyridine-induced hyperexcitability (Colbert and Pan, 1999). These findings suggest that Kv2.1 can indeed act as a potent suppressor of neuronal hyperexcitability. Together with the results presented here, we suggest that, like $\mathrm{K}_{\mathrm{ATP}}$ and $\mathrm{BK}$ channels, Kv2.1 can also couple metabolic state and $\left[\mathrm{Ca}^{2+}\right]_{\mathrm{i}}$ to membrane excitability under periods of hypoxia/ischemia. However, for Kv2.1, such coupling occurs via a more indirect and slower mechanism than for the intrinsically ATP- and $\mathrm{Ca}^{2+}$. sensitive $\mathrm{K}^{+}$channels, acting through dynamic and reversible calcineurin-dependent changes in phosphorylation state of the channel protein (supplemental Fig. 4, available at www.jneurosci.org as supplemental material). Given the extremely high level of Kv2.1 expression in the somata and proximal dendrites of hippocampal (and virtually all other central) neurons, this $\mathrm{Kv}$ channel may represent a key but previously unrecognized contributor to the metabolic state- and $\mathrm{Ca}^{2+}$-dependent regulation of neuronal excitability.

\section{Short- versus long-term effects of Kv2.1 modulation}

Given the dynamic regulation of Kv2.1 function by reversible changes in phosphorylation state, it is not surprising that the role of $\mathrm{Kv} 2.1 / I_{\mathrm{K}}$ in neuroprotection is complex. Increases in delayed rectifier $\mathrm{K}^{+}$channel activity in response to severe hypoxia and ischemia have been observed previously in CA1 hippocampal neurons (Chi and $\mathrm{Xu}, 2000,2001$; Zou et al., 2005), in large aspiny neostriatal neurons (Deng et al., 2005), in substantia nigra neurons (Jiang and Haddad, 1993), and in dorsal vagal motor neurons (Cowan and Martin, 1992). In cultured cortical neurons, increased TEA-sensitive $I_{\mathrm{K}}$ in response to proapoptotic treatments (long-term serum deprivation or staurosporine treatment) is critical to the induction of apoptosis, such that TEA is antiapoptotic (Yu et al., 1997, 1999b; Wang et al., 2000; Wei et al., 2003). Models linking $I_{\mathrm{K}^{-}}$mediated $\mathrm{K}^{+}$efflux to altered $\left[\mathrm{K}^{+}\right]_{\mathrm{i}}$ homeostasis, cell shrinkage, and proapoptotic cytochrome $c$ release from mitochondria, as well as to direct $\mathrm{K}^{+}$regulation of caspase, have been proposed (Yu et al., 1999b; Wei et al., 2003; Hribar et al., 2004). Knockdown experiments in cultured cortical neurons revealed that Kv2.1 is the specific TEA-sensitive $\mathrm{Kv}$ channel underlying the proapoptotic $I_{\mathrm{K}}$ activity (Pal et al., 2003). However, we and others (Katsuki et al., 2005) found that TEA treatment exacerbated, not attenuated, neuronal injury induced by brief ischemia. Differences in the severity of insults and the modes of cell death involved, as well as the specific experimental system under study, may impact the effects of $I_{K} / \mathrm{Kv} 2.1$ modulation on neuronal survival. The bulk of the previous studies (Yu et al., 1997, 1999a,b; Pal et al., 2003; Wei et al., 2003) showing proapoptotic roles for $I_{\mathrm{K}} / \mathrm{Kv} 2.1$ exposed neurons to relatively long (several hours to days) insults to cause extensive and sustained $\mathrm{K}^{+}$efflux, cell shrinkage, and neuronal apoptosis. In our in vitro ischemia model, acute and brief CI $(\sim 15 \mathrm{~min})$ in itself was not sufficient to kill neurons but together with TEA treatment in- 
duced hyperexcitability and cell death, presumably by an excitotoxic non-apoptotic pathway. Because brain ischemia can kill neurons via both apoptosis and necrosis (Zhang et al., 2004), the complex neuroprotective and/or proapoptotic roles proposed for $I_{\mathrm{K}} / \mathrm{Kv} 2.1$ may also reflect Kv2.1 modulation in these different contexts. Our results are also consistent with a previous study that TEA does not protect against necrotic neuronal death induced by brief ischemic insult but provides neuroprotection against apoptotic cell death induced by longer, more intense insults (Wei et al., 2003). In addition, the timing of TEA addition relative to that of the ischemic insult can dramatically impact the neuroprotective effects of TEA in vivo (Huang et al., 2001).

We suggest that Kv2.1 might have a dual role in response to neuronal insults: an immediate protective role against acute hyperexcitability induced by seizures (Misonou et al., 2004) and brief $(\sim 2-15 \mathrm{~min})$ hypoxia attributable to calcineurindependent dephosphorylation, and altered localization and function, leading to homeostatic suppression of neuronal hyperexcitability. Removal of the ischemic (or excitotoxic) insult leads to a dynamic reversal of the calcineurin-dependent dephosphorylation and a recovery of the normal Kv2.1 phosphorylation state, clustering, and voltage-dependent activation. However, sustaining Kv2.1 in the dephosphorylated state under conditions of prolonged insult may lead to excessive $\mathrm{K}^{+}$efflux, cell shrinkage, and apoptotic cell death. Thus, the role of Kv2.1 in neuronal survival in response to ischemic and other insults may be biphasic (supplemental Fig. 4, available at www.jneurosci.org as supplemental material). Brief and reversible activation of Kv2.1 by dephosphorylation may be advantageous attributable to neuroprotective suppression of neuronal excitability, but sustained activation may eventually lead to deleterious effects via lowered $\left[\mathrm{K}^{+}\right]_{\mathrm{i}}$.

\section{Possible mechanisms of ischemia-induced modulation of $\mathrm{Kv} 2.1$}

Electron microscopic analysis has shown that plasma membrane Kv2.1 clusters on the somata and proximal dendrites of pyramidal neurons lie over subsurface cisternae (Du et al., 1998), intracellular ER-derived membranes rich in inositol triphosphate receptors and ryanodine receptors, and associated with high concentrations of mitochondria (Berridge, 1998; Paspalas and Goldman-Rakic, 2004). We found that, in cultured hippocampal neurons plasma membrane, Kv2.1 clusters overlap clusters of ryanodine receptors and the luminal $\mathrm{Ca}^{2+}$-binding protein calsequestrin (Antonucci et al., 2001; Misonou et al., 2005), suggesting a specialized neuronal $\mathrm{Ca}^{2+}$ signaling domain that may also contain a high density of calcineurin (Cameron et al., 1995). That FCCP, but not thapsigargin and caffeine, mimicked the effects of CI on modulation of Kv2.1, and that FCCP and CI treatments together yielded no additional increase in $\left[\mathrm{Ca}^{2+}\right]_{\mathrm{i}}$ than achieved with CI alone suggest that mitochondria, especially ERassociated mitochondria (Berridge, 1998; Paspalas and Goldman-Rakic, 2004), are good candidates for the source of rapid intracellular $\mathrm{Ca}^{2+}$ release induced by ischemia. A number of recent studies have revealed the critical role for neuronal mitochondria in regulating $\mathrm{Ca}^{2+}$-dependent signaling events (Yang et al., 2003; Li et al., 2004). Moreover, mitochondrial function can be disturbed under such pathological conditions, for example, $\mathrm{Ca}^{2+}$ is released from mitochondria through the $2 \mathrm{Na}^{+} / \mathrm{Ca}^{2+}$ exchanger in hippocampal neurons during oxygen-glucose deprivation (Zhang and Lipton, 1999). $\mathrm{Ca}^{2+}$ stores in the cistern itself are an additional source of CI-induced $\mathrm{Ca}^{2+}$ release, as occurs in ischemic spinal cord neurons (Ouardouz et al., 2003). The localization of Kv2.1 at sites close to subsurface cisterns and associated mitochondria may allow for more efficient functional coupling of $\mathrm{Ca}^{2+}$ released from these intracellular stores to modulation of plasma membrane Kv2.1 channels. Moreover, such assemblies may allow for local "cluster-by-cluster" or quantal regulation of discrete pools of Kv2.1 in a manner distinct from the more graded response expected for a more uniformly distributed channel population. Dispersion of these Kv2.1 clusters during calcineurin-mediated dephosphorylation could provide a mechanism for more efficient reversibility and recovery, by removing Kv2.1 from this region of local $\mathrm{Ca}^{2+}$ and calcineurin signaling. The coupling of neuronal metabolism and $\left[\mathrm{Ca}^{2+}\right]_{\mathrm{i}}$ to dynamic changes in Kv2.1 localization and function via regulation of the Kv2.1 phosphorylation state provides an effective and reversible mechanism for homeostatic regulation of central neuron excitability under conditions of ischemic and excitotoxic stress.

\section{References}

Antonucci DE, Lim ST, Vassanelli S, Trimmer JS (2001) Dynamic localization and clustering of dendritic Kv2.1 voltage-dependent potassium channels in developing hippocampal neurons. Neuroscience 108:69-81.

Ballanyi K (2004) Protective role of neuronal KATP channels in brain hypoxia. J Exp Biol 207:3201-3212.

Bekkers JM (2000) Distribution and activation of voltage-gated potassium channels in cell-attached and outside-out patches from large layer 5 cortical pyramidal neurons of the rat. J Physiol (Lond) 525:611-620.

Bernstein BW, Bamburg JR (2003) Actin-ATP hydrolysis is a major energy drain for neurons. J Neurosci 23:1-6.

Berridge MJ (1998) Neuronal calcium signaling. Neuron 21:13-26.

Calabresi P, Cupini LM, Centonze D, Pisani F, Bernardi G (2003) Antiepileptic drugs as a possible neuroprotective strategy in brain ischemia. Ann Neurol 53:693-702.

Cameron AM, Steiner JP, Roskams AJ, Ali SM, Ronnett GV, Snyder SH (1995) Calcineurin associated with the inositol 1,4,5-trisphosphate receptor-FKBP12 complex modulates $\mathrm{Ca}^{2+}$ flux. Cell 83:463-472.

Chi XX, Xu ZC (2000) Differential changes of potassium currents in CA1 pyramidal neurons after transient forebrain ischemia. J Neurophysiol $84: 2834-2843$.

Chi XX, Xu ZC (2001) Alterations of single potassium channel activity in CA1 pyramidal neurons after transient forebrain ischemia. Neuroscience 108:535-540.

Chow E, Haddad GG (1998) Differential effects of anoxia and glutamate on cultured neocortical neurons. Exp Neurol 150:52-59.

Colbert CM, Pan E (1999) Arachidonic acid reciprocally alters the availability of transient and sustained dendritic $\mathrm{K}^{+}$channels in hippocampal CA1 pyramidal neurons. J Neurosci 19:8163-8171.

Cowan AI, Martin RL (1992) Ionic basis of membrane potential changes induced by anoxia in rat dorsal vagal motoneurones. J Physiol (Lond) 455:89-109.

Deng P, Pang ZP, Zhang Y, Xu ZC (2005) Increase of delayed rectifier potassium currents in large aspiny neurons in the neostriatum following transient forebrain ischemia. Neuroscience 131:135-146.

Du J, Tao-Chang J-H, Zerfas P, McBain CJ (1998) The $\mathrm{K}^{+}$channel, Kv2.1, is apposed to astrocytic processes and is associated with inhibitory postsynaptic membranes in hippocampal and cortical principal neurons and inhibitory interneurons. Neuroscience 84:37-48.

Du J, Haak LL, Phillips-Tansey E, Russell JT, McBain CJ (2000) Frequencydependent regulation of rat hippocampal somato-dendritic excitability by the $\mathrm{K}^{+}$channel subunit Kv2.1. J Physiol (Lond) 522:19-31.

Erdemli G, Xu YZ, Krnjevic K (1998) Potassium conductance causing hyperpolarization of CA1 hippocampal neurons during hypoxia. J Neurophysiol 80:2378-2390.

Griesemer D, Zawar C, Neumcke B (2002) Cell-type specific depression of neuronal excitability in rat hippocampus by activation of ATP-sensitive potassium channels. Eur Biophys J 31:467-477.

Hribar M, Bloc A, Medilanski J, Nusch L, Eder-Colli L (2004) Voltage-gated $\mathrm{K}^{+}$current: a marker for apoptosis in differentiating neuronal progenitor cells? Eur J Neurosci 20:635-648.

Huang H, Gao TM, Gong L, Zhuang Z, Li X (2001) Potassium channel 
blocker TEA prevents CA1 hippocampal injury following transient forebrain ischemia in adult rats. Neurosci Lett 305:83-86.

Immke D, Wood M, Kiss L, Korn SJ (1999) Potassium-dependent changes in the conformation of the Kv2.1 potassium channel pore. J Gen Physiol 113:819-836.

Jiang C, Haddad GG (1993) Short periods of hypoxia activate a $\mathrm{K}^{+}$current in central neurons. Brain Res 614:352-356.

Kang J, Huguenard JR, Prince DA (2000) Voltage-gated potassium channels activated during action potentials in layer $\mathrm{V}$ neocortical pyramidal neurons. J Neurophysiol 83:70-80.

Katsuki H, Shinohara A, Fujimoto S, Kume T, Akaike A (2005) Tetraethylammonium exacerbates ischemic neuronal injury in rat cerebrocortical slice cultures. Eur J Pharmacol 508:85-91.

Kawasaki K, Traynelis SF, Dingledine R (1990) Different responses of CA1 and CA3 regions to hypoxia in rat hippocampal slice. J Neurophysiol 63:385-394.

Korngreen A, Sakmann B (2000) Voltage-gated $\mathrm{K}^{+}$channels in layer 5 neocortical pyramidal neurones from young rats: subtypes and gradients. J Physiol (Lond) 525:621-639.

Lanni F, Wilson T (2000) Grating image systems or optical sectioning fluorescence microscopy of cells, tissues, and small organisms. Cold Spring Harbor, NY: Cold Spring Harbor Laboratory.

Lee JM, Zipfel GJ, Choi DW (1999) The changing landscape of ischaemic brain injury mechanisms. Nature 399:A7-A14.

Leker RR, Neufeld MY (2003) Anti-epileptic drugs as possible neuroprotectants in cerebral ischemia. Brain Res Brain Res Rev 42:187-203.

Li Z, Okamoto K, Hayashi Y, Sheng M (2004) The importance of dendritic mitochondria in the morphogenesis and plasticity of spines and synapses. Cell 119:873-887.

Lim ST, Antonucci DE, Scannevin RH, Trimmer JS (2000) A novel targeting signal for proximal clustering of the $\mathrm{Kv} 2.1 \mathrm{~K}^{+}$channel in hippocampal neurons. Neuron 25:385-397.

Malin SA, Nerbonne JM (2002) Delayed rectifier $\mathrm{K}^{+}$currents, IK, are encoded by Kv2 $\alpha$-subunits and regulate tonic firing in mammalian sympathetic neurons. J Neurosci 22:10094-10105.

Martina M, Schultz JH, Ehmke H, Monyer H, Jonas P (1998) Functional and molecular differences between voltage-gated $\mathrm{K}^{+}$channels of fastspiking interneurons and pyramidal neurons of rat hippocampus. J Neurosci 18:8111-8125.

Misonou H, Mohapatra DP, Park EW, Leung V, Zhen D, Misonou K, Anderson AE, Trimmer JS (2004) Regulation of ion channel localization and phosphorylation by neuronal activity. Nat Neurosci 7:711-718.

Misonou H, Mohapatra DP, Trimmer JS (2005) Kv2.1: a voltage-gated $\mathrm{K}^{+}$ channel critical to dynamic control of neuronal excitability. Neurotoxicology 26:743-752.

Muennich EA, Fyffe RE (2003) Focal aggregation of a voltage-gated potassium channel, Kv2.1, at synaptic sites in rat spinal motoneurons. J Physiol (Lond) 554:673-685.

Murakoshi H, Trimmer JS (1999) Identification of the Kv2.1 $\mathrm{K}^{+}$channel as a major component of the delayed rectifier $\mathrm{K}^{+}$current in rat hippocampal neurons. J Neurosci 19:1728-1735.

Murakoshi H, Shi G, Scannevin RH, Trimmer JS (1997) Phosphorylation of the Kv2.1 K ${ }^{+}$channel alters voltage-dependent activation. Mol Pharmacol 52:821-828.

Namba C, Adachi N, Liu K, Yorozuya T, Arai T (2002) Suppression of sodium pump activity and an increase in the intracellular $\mathrm{Ca}^{2+}$ concentration by dexamethasone in acidotic mouse brain. Brain Res 957:271-277.

Ouardouz M, Nikolaeva MA, Coderre E, Zamponi GW, McRory JE, Trapp BD, Yin X, Wang W, Woulfe J, Stys PK (2003) Depolarization-induced $\mathrm{Ca}^{2+}$ release in ischemic spinal cord white matter involves L-type $\mathrm{Ca}^{2+}$ channel activation of ryanodine receptors. Neuron 40:53-63.

Pal S, Hartnett KA, Nerbonne JM, Levitan ES, Aizenman E (2003) Mediation of neuronal apoptosis by Kv2.1-encoded potassium channels. J Neurosci 23:4798-4802.
Paspalas CD, Goldman-Rakic PS (2004) Microdomains for dopamine volume neurotransmission in primate prefrontal cortex. J Neurosci 24:5292-5300.

Rhodes KJ, Carroll KI, Sung MA, Doliveira LC, Monaghan MM, Burke SL, Strassle BW, Buchwalder L, Menegola M, Cao J, An WF, Trimmer JS (2004) KChIPs and Kv4 $\alpha$ subunits as integral components of A-type potassium channels in mammalian brain. J Neurosci 24:7903-7915.

Runden-Pran E, Haug FM, Storm JF, Ottersen OP (2002) BK channel activity determines the extent of cell degeneration after oxygen and glucose deprivation: a study in organotypical hippocampal slice cultures. Neuroscience 112:277-288.

Shi G, Kleinklaus AK, Marrion NV, Trimmer JS (1994) Properties of Kv2.1 $\mathrm{K}^{+}$channels expressed in transfected mammalian cells. J Biol Chem 269:23204-23211.

Surmeier DJ, Foehring R (2004) A mechanism for homeostatic plasticity. Nat Neurosci 7:691-692.

Swanson RA, Benington JH (1996) Astrocyte glucose metabolism under normal and pathological conditions in vitro. Dev Neurosci 18:515-521.

Tanabe M, Mori M, Gahwiler BH, Gerber U (1999) Apamin-sensitive conductance mediates the $\mathrm{K}^{+}$current response during chemical ischemia in CA3 pyramidal cells. J Neurophysiol 82:2876-2882.

Trimmer JS (1991) Immunological identification and characterization of a delayed rectifier $\mathrm{K}^{+}$channel polypeptide in rat brain. Proc Natl Acad Sci USA 88:10764-10768.

Trimmer JS, Rhodes KJ (2004) Localization of voltage-gated ion channels in mammalian brain. Annu Rev Physiol 66:477-519.

Varelas PN, Mirski MA (2001) Seizures in the adult intensive care unit. J Neurosurg Anesthesiol 13:163-175.

Wang X, Xiao AY, Ichinose T, Yu SP (2000) Effects of tetraethylammonium analogs on apoptosis and membrane currents in cultured cortical neurons. J Pharmacol Exp Ther 295:524-530.

Wei L, Yu SP, Gottron F, Snider BJ, Zipfel GJ, Choi DW (2003) Potassium channel blockers attenuate hypoxia- and ischemia-induced neuronal death in vitro and in vivo. Stroke 34:1281-1286.

Yamada K, Ji JJ, Yuan H, Miki T, Sato S, Horimoto N, Shimizu T, Seino S, Inagaki N (2001) Protective role of ATP-sensitive potassium channels in hypoxia-induced generalized seizure. Science 292:1543-1546.

Yamamoto S, Tanaka E, Higashi H (1997) Mediation by intracellular calcium-dependent signals of hypoxic hyperpolarization in rat hippocampal CA1 neurons in vitro. J Neurophysiol 77:386-392.

Yang F, He XP, Russell J, Lu B (2003) $\mathrm{Ca}^{2+}$ influx-independent synaptic potentiation mediated by mitochondrial $\mathrm{Na}^{+}-\mathrm{Ca}^{2+}$ exchanger and protein kinase C. J Cell Biol 163:511-523.

Yu SP, Yeh CH, Sensi SL, Gwag BJ, Canzoniero LM, Farhangrazi ZS, Ying HS, Tian M, Dugan LL, Choi DW (1997) Mediation of neuronal apoptosis by enhancement of outward potassium current. Science 278:114-117.

Yu SP, Yeh C, Strasser U, Tian M, Choi DW (1999a) NMDA receptormediated $\mathrm{K}^{+}$efflux and neuronal apoptosis. Science 284:336-339.

Yu SP, Yeh CH, Gottron F, Wang X, Grabb MC, Choi DW (1999b) Role of the outward delayed rectifier $\mathrm{K}^{+}$current in ceramide-induced caspase activation and apoptosis in cultured cortical neurons. J Neurochem 73:933-941.

Zawar C, Neumcke B (2000) Differential activation of ATP-sensitive potassium channels during energy depletion in CA1 pyramidal cells and interneurones of rat hippocampus. Pflügers Arch 439:256-262.

Zhang F, Yin W, Chen J (2004) Apoptosis in cerebral ischemia: executional and regulatory signaling mechanisms. Neurol Res 26:835-845.

Zhang Y, Lipton P (1999) Cytosolic $\mathrm{Ca}^{2+}$ changes during in vitro ischemia in rat hippocampal slices: major roles for glutamate and $\mathrm{Na}^{+}$-dependent $\mathrm{Ca}^{2+}$ release from mitochondria. J Neurosci 19:3307-3315.

Zou B, Li Y, Deng P, Xu ZC (2005) Alterations of potassium currents in ischemia-vulnerable and ischemia-resistant neurons in the hippocampus after ischemia. Brain Res 1033:78-89. 